\title{
Multispectral UAV monitoring of submerged seaweed in shallow water
}

\author{
Yuri Taddia $^{1}$ (D) Paolo Russo $^{1} \cdot$ Stefano Lovo $^{2} \cdot$ Alberto Pellegrinelli $^{1}$
}

Received: 29 November 2018 / Accepted: 5 May 2019 / Published online: 22 May 2019

(C) The Author(s) 2019

\begin{abstract}
The sudden algal bloom in shallow water may be a serious problem for sea coastal economy based on clams farming because it leads quickly to anoxia conditions with the consequent death of the molluscs. In order to detect the rise of algae, normally satellite remote sensing is used, exploiting the higher response in the near infrared wavelengths. A recent progress in monitoring this phenomenon derives from the availability of unmanned aerial vehicles (UAVs) equipped with lightweight multispectral cameras. Such technique makes it possible to acquire detailed spectral information with narrow bands attaining an assessment of the algal bloom at both high geometric and radiometric resolutions. In this work, we tested the MicaSense RedEdge-M multispectral camera mounted on a DJI Phantom 3 Professional aircraft to map submerged seaweeds and assess their evolution with particular regard to the importance of the radiometric calibration of raw imageries using a Downwelling Light Sensor (DLS) and a known reflectance panel. The case study is the lagoon of Goro (Northern Adriatic Sea, Italy), a crucial environment for the clams farming in the Emilia-Romagna region. Digital images acquired in two subsequent flights were processed with either Agisoft PhotoScan Professional and Pix4D Mapper Pro varying the calibration strategies. After a pre-analysis, we applied two different approaches for the seaweed detection: NDVI and maximum likelihood classification. All the tests performed in this study confirm that the monitoring over time with a multispectral lightweight camera mounted on a UAV is possible, but also that by applying proper radiometric corrections, most accurate and reliable results can be achieved.
\end{abstract}

Keywords Unmanned aerial vehicles (UAVs) · Multispectral camera · Coastal lagoon environment · Radiometric calibration · Submerged seaweed monitoring

\section{Introduction}

In the last few years, an increasing eutrophication of the seaweed occurred in the northern Adriatic Sea, especially near the

Yuri Taddia

yuri.taddia@unife.it

Paolo Russo

paolo.russo@unife.it

Stefano Lovo

slovo@arpae.it

Alberto Pellegrinelli

alberto.pellegrinelli@unife.it

1 Engineering Department, University of Ferrara, via Saragat 1, 44122 Ferrara, Italy

2 Regional Agency for Prevention, Environment and Energy of Emilia-Romagna (Arpae)-Oceanographic Structure Daphne, via Bologna 534, 44124 Ferrara, Italy
Po River delta. The fresh water coming from the river mouths, in fact, supplies a high content of nutrients for the algal growth. During the summer, even a limited increase of the seawater temperature can suddenly lead to an uncontrolled bloom with harmful effects on fish and aquatic life (Hallegraeff 2003).

Regions where mollusk cultures are present appear to be particularly vulnerable by the anoxia conditions that can occur whenever the biomass of the seaweed starts the decomposition process.

The economy of most of the towns located around the Po River delta strongly depends on the fisheries and the aquaculture and is therefore threatened by the eutrophication.

The Regional Agency for Prevention, Environment and Energy of Emilia-Romagna (Arpae) constantly monitors strategical areas. Recognitions are periodically performed by the means of boats in search of the presence of emerging seaweed. In addition, the water quality is assessed by the deployment of environmental probes collecting data about the temperature, the salinity, $\mathrm{pH}$, and the oxygen content of the seawater. 
Despite this effort that allows to potentially foresee the occurrence of conditions conducive to the development of the seaweed, the early monitoring of the most affected areas is particularly difficult.

In fact, the spatial distribution of the zones where the seaweed growth is higher varies over the years. Therefore, only a monitoring starting from the initial stage when the seaweed is still completely submerged may enable to make reliable predictions and to effectively respond in case of the development of harmful conditions.

Satellite multispectral images are still the primary source of remote sensing data for seaweed mapping, but the limited number of spectral bands (less than ten) and the frequent occurrence of mixed pixel (also for medium-high resolution geometric satellites, in the range of $1 \div 5 \mathrm{~m} / \mathrm{pixel}$ ), limit the possibility of an accurate seaweed monitoring (Hossain et al. 2015; Wicaksono et al. 2017).

The recent developments in Unmanned Aerial Vehicles (UAVs) technology with miniaturization of sensors increase the flight capabilities and agility of UAVs. Furthermore, the high-quality imagery combined with a structure from motion ( $\mathrm{SfM})$ approach has cleared the way to UAV-based research applications. Accurate digital surface or terrain models, contours, textured 3D models, and 3D mapping are examples of typical SfM final products (Nex and Remondino 2014). UAVs coupled with multispectral imagers at $\mathrm{cm}$-level resolution offer a wide range of possibilities in precision farming and forestry management (Candiago et al. 2015). With regard to the marine field, coastline zone identification (Papakonstantinou et al. 2016), 2D/3D coastal environment characterization (Mancini et al. 2013; Taddia et al. 2019), and seaweed monitoring or algal bloom research (Kislik et al. 2018; Duffy et al. 2018) are further applications of UAV-borne imageries.

Thanks to the benefits in using UAVs in environmental applications, in our research, we decided to test the use of a low-cost multispectral system made of a DJI Phantom 3 Professional equipped with the multispectral camera MicaSense RedEdge-M for the detection of the submerged seaweed. The mapping was performed in a lagoon environment characterized by shallow and sheltered waters with a tidal excursion limited to few tens of centimeters. In such conditions, the use of UAVs could be particularly indicated.

In order to compute actual reflectance values from the digital numbers on the raw imageries, the radiometric calibration represents a fundamental task. Recently, many articles have focused on the importance of the radiometric calibration of multispectral and hyperspectral data (Hakala et al. 2018; Honkavaara and Khoramshahi 2018; Deng et al. 2018b). The computation of at-sensor radiance represents the first step in converting a digital number into a reflectance. This conversion, which is performed accounting for dark current, ISO sensitivity, exposure time, and vignetting (von Bueren et al. 2015; Aasen et al. 2018; MicaSense 2018), allows to evaluate the actual flux of energy that has been detected by means of the multispectral sensor. The subsequent computation of the surface's reflectance may be performed by both using Downwelling Light Sensor's (DLS) irradiance measurements or by the means of a known reflectance panel. Hakala et al. (2018) proposed a method for the direct reflectance measurement by UAV platforms, exploiting both at-sensor radiance measurements carried out by a hyperspectral camera and irradiance measurements performed using a spectrometer. However, in their research, the authors highlight how a tilt of the irradiance sensor of few degrees may drastically affect the accuracy in the assessment of the actual irradiance. Known reflectance panels are able to address this kind of issues.

The reconstruction of spectral information at pixel level necessarily involves a registration of the images acquired by the different optics of the multispectral sensor. Issues related to the fine band-to-band registration of such multispectral imageries acquired by $2 \mathrm{D}$ imagers with a multiple camera array have been studied by Jhan et al. (2016) and finally addressed by proposing an ortho-rectification procedure.

Nowadays, the most common approach for managing multispectral datasets consists in using structure from motion techniques to generate a comprehensive orthomosaic for each band. However, a same object may be reconstructed by having different reflectance values on multiple images. Therefore, Honkavaara and Khoramshahi (2018) investigated an approach to overcome the inconsistent reflectance values that may be computed in overlapping regions of UAV-acquired images, proposing a global optimization approach for the radiometric correction of an entire image block.

Deng et al. (2018a) have also investigated the effect of the spatial resolution on the performance of both radiometric and geometric acquisitions with UAV-borne hyperspectral imagers. Especially for known reflectance panels, the surroundings may influence the detected response of the panel used as a reference standard. Consequently, the authors have proposed methods for an appropriate selection of the spatial resolution by UAVs in order to improve the operational efficiency. However, in our research, the images of the known reflectance panel were always acquired not during the flight, but instead holding the multispectral camera directly above the panel at no more than $1-\mathrm{m}$ distance just before and just after each flight.

In spite of advanced radiometric calibration methods that have been proposed in the literature, such the ones mentioned above, in our work, we used a simplified overall data processing. In particular, we used two of the most common SfM software to stitch all the images (i.e., Agisoft PhotoScan Professional and Pix4D Mapper Pro). Both are capable to apply radiometric corrections based on DLS data and images of a known reflectance panel. Consequently, we also 
Fig. 1 The site of the lagoon of Goro, in the Northern Adriatic Sea, Italy; site 1 and site 2 represent the locations where the surveys were carried out

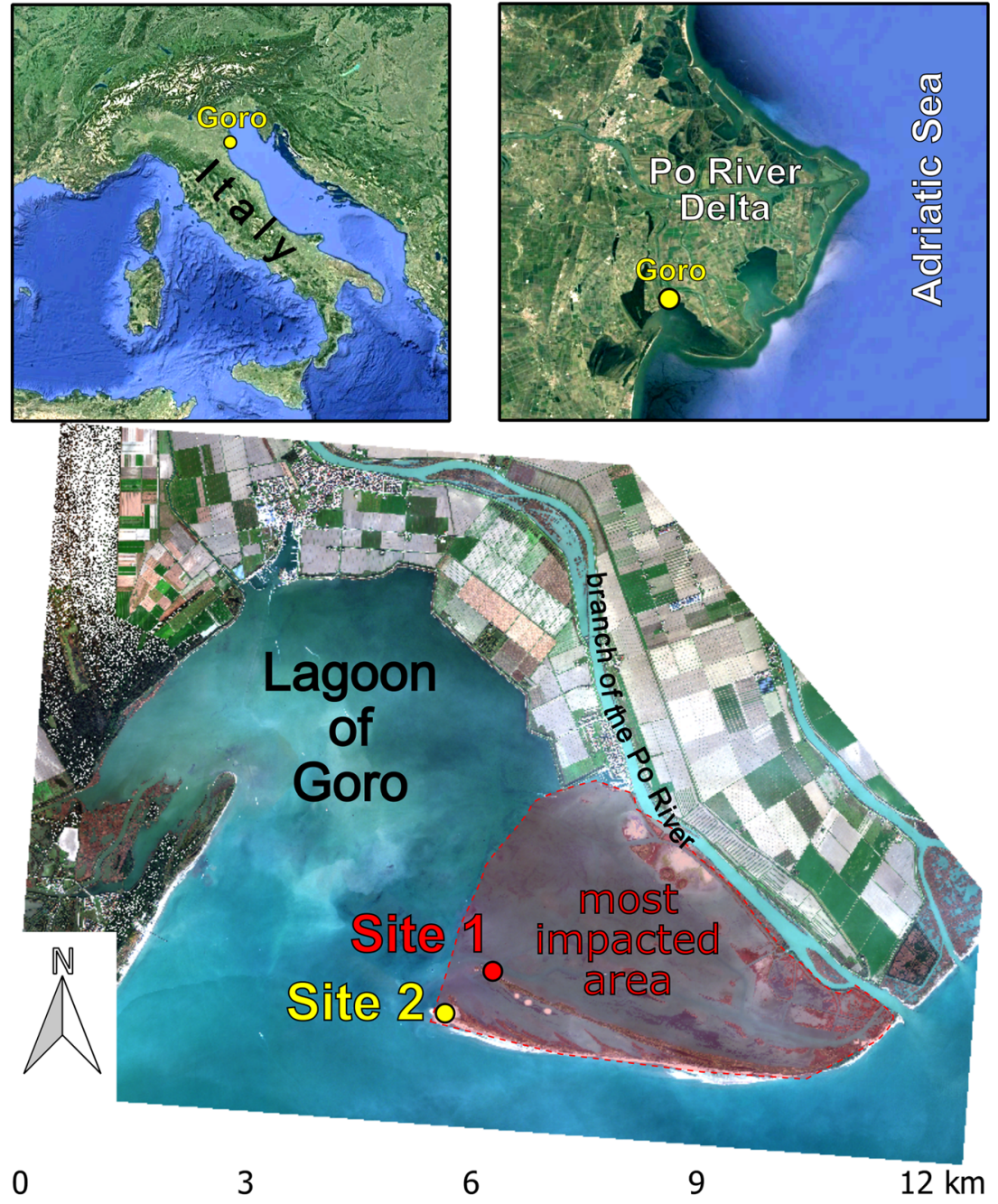

0

3

orthomosaics) by both software as a secondary purpose of the research.

After this preliminary assessment, necessary to investigate potential differences due to the data processing software, we proceeded to the detection of the submerged seaweed.

The quantification was hence performed through two different techniques:

- a simple spectral index computation, i.e., the Normalized Difference Vegetation Index (NDVI);

- a supervised classification conducted by the means of a standard pixel-based algorithm, well known in the field of remote sensing: maximum likelihood (Richards and Xiuping 2006). This procedure was performed in ENVI software.

The supervised classification provided more reliable results than the simple spectral index computation and proved to be applicable to wider regions, especially to those where variations in water depth were higher.
Fig. 2 Submerged and emerged seaweed in shallow water (site 1 in Fig. 1) 
Fig. 3 The MicaSense RedEdge$\mathrm{M}$ multispectral camera (a) mounted on the DJI Phantom 3 Professional. The Downwelling Light Sensor (DLS) is set up on the top of the aircraft (b)

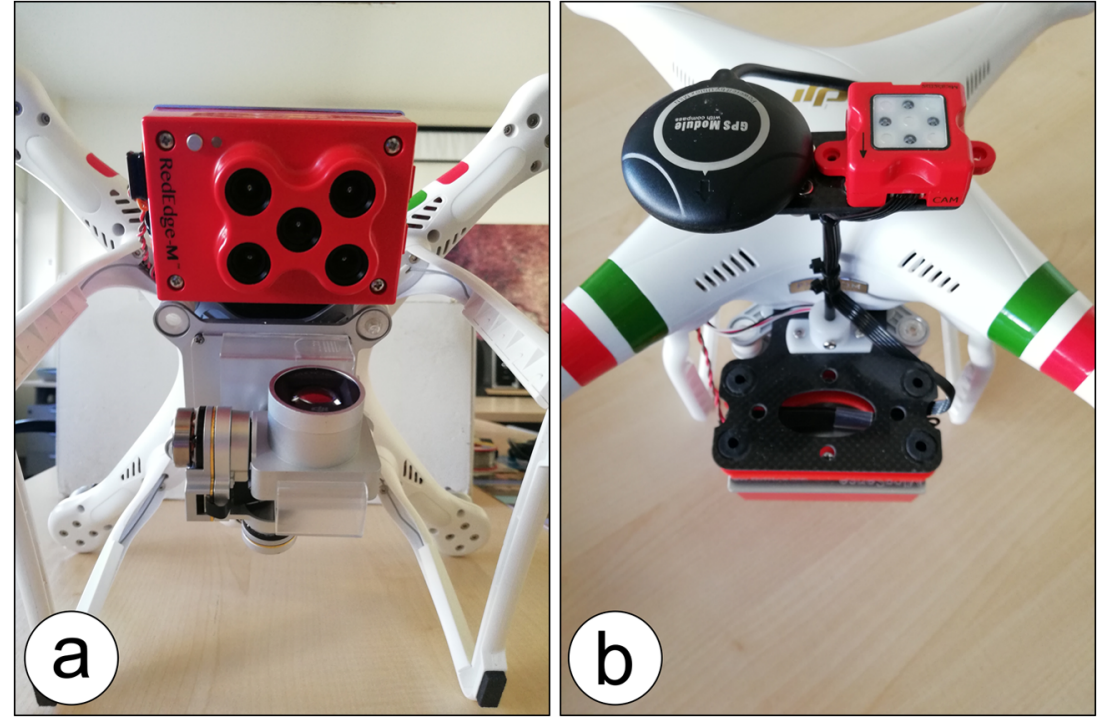

Finally, the last investigation performed within this research, upon the successful quantification and monitoring over time of the submerged seaweed extents, consisted in evaluating whether a supervised classification may be sufficient to overcome the lack of a radiometric calibration of the raw imageries.

Table 1 MicaSense RedEdge-M multispectral camera specifications. (FWHM is the full width at half maximum)

MicaSense RedEdge-M specifications

\begin{tabular}{|c|c|c|}
\hline Weight & \multicolumn{2}{|c|}{$170 \mathrm{~g}$ including DLS } \\
\hline Dimensions & \multicolumn{2}{|c|}{$94 \mathrm{~mm} \times 63 \mathrm{~mm} \times 46 \mathrm{~mm}$} \\
\hline Power & \multicolumn{2}{|c|}{$4.2 \mathrm{~V}-15.8 \mathrm{~V}, 4 \mathrm{~W}$ nominal, $8 \mathrm{~W}$ peak } \\
\hline \multirow[t]{5}{*}{ Spectral bands } & Blue & $\begin{array}{l}\text { Center wavelength: } \\
475 \mathrm{~nm} \\
\text { Bandwith FWHM: } \\
20 \mathrm{~nm}\end{array}$ \\
\hline & Green & $\begin{array}{l}\text { Center wavelength: } \\
560 \mathrm{~nm} \\
\text { Bandwith FWHM: } \\
20 \mathrm{~nm}\end{array}$ \\
\hline & Red & $\begin{array}{l}\text { Center wavelength: } \\
668 \mathrm{~nm} \\
\text { Bandwith FWHM: } \\
10 \mathrm{~nm}\end{array}$ \\
\hline & Red Edge & $\begin{array}{l}\text { Center wavelength: } \\
717 \mathrm{~nm} \\
\text { Bandwith FWHM } 10 \mathrm{~nm}\end{array}$ \\
\hline & Near IR & $\begin{array}{l}\text { Center wavelength: } \\
840 \mathrm{~nm} \\
\text { Bandwith FWHM: } 40 \mathrm{~nm}\end{array}$ \\
\hline Ground sample distance (GSD) & $8 \mathrm{~cm}$ at 12 & $0 \mathrm{~m}(\approx 5 \mathrm{~cm}$ at $70 \mathrm{~m})$ \\
\hline
\end{tabular}

\section{The case study}

The lagoon of Goro (Fig. 1) is located in the Northern Adriatic Sea (Italy) and it is part of the Po River delta. This particular environment is characterized by shallow water (up to $1.5 \mathrm{~m}$ ) and high hydro-dynamics, with a continuous shoreline evolution due to the sand transportation (Corbau et al. 2016).

Satellite imageries (Quickbird and WorldView-2) characterized by a geometric resolution up to half-metre level have been used in the last years for the accurate mapping of this phenomenon.

Thanks to both the shallow water and the overall environmental conditions typical of any lagoon, this coastal section adjacent to the town of Goro is particularly suitable for the mollusk farming, especially concerning the clams. For the local community, this represents the primary economic activity.

During the summer, especially in the most impacted region highlighted in Fig. 1, the sudden and practically uncontrolled algal bloom, facilitated by a warmer water, may rapidly lead to anoxia conditions that are harmful for the clams. To prevent this dreadful situation, a constant monitoring activity is conducted by the use of boats in search for emerged seaweed. This effort is enforced whenever the temperature and the salinity of the water become higher than usual.

However, the presence of emerged seaweed is a clear sign of an algal bloom in an already advanced status (Fig. 2). To avoid the further occurrence of anoxia conditions in those situations, it is necessary to take promptly countermeasures such as the removal of those emerged algae before they rot.

The prevention of this phenomenon by means of an adequate monitoring technique and the performance of effective actions to improve the water quality would hence be the more appropriate approach. 
Table 2 Advantages and disadvantages of each radiometric calibration method

\begin{tabular}{lll}
\hline Method & Advantages & Disadvantages \\
\hline DLS & Irradiance measurement in Exif metadata. & Reflectance is not much accurate. \\
Known reflectance panel & Reflectance is very accurate. & Operators must remember to acquire panel's image(s). \\
& No irradiance variations may be addressed. \\
DLS + known reflectance panel & $\begin{array}{l}\text { Reflectance is very accurate. } \\
\text { Irradiance variations are addressed. }\end{array}$ & \\
\hline
\end{tabular}

The detection of the submerged seaweed (Fig. 2) extents can represent the solution for both saving time and preventing the occurrence of harmful water conditions.

\section{Materials and methods}

The recent deployment of UAVs has involved an increasing variety of applications in many fields. With regard to survey, their use in photogrammetry proved to be effective in terms of both geometric resolution and accuracy, as well as providing a system with an affordable cost. On the base of RGB images captured through a specially planned mission, the structure from motion techniques enable to accurately reconstruct the geometry of the surveyed object.

Moreover, the availability of lightweight multispectral cameras makes it possible to extend the spectral information collectable by UAVs to the infrared region. This capability is really crucial because most of the vegetation, including the seaweed, have higher response in the near infrared (around $840 \mathrm{~nm}$ ) and in the so-called "red edge" (around $717 \mathrm{~nm}$ ).

In order to monitor the algal growth, a proximity multispectral approach has been adopted by means of a DJI Phantom 3 Professional aircraft equipped with a MicaSense RedEdge-M (Fig. 3).

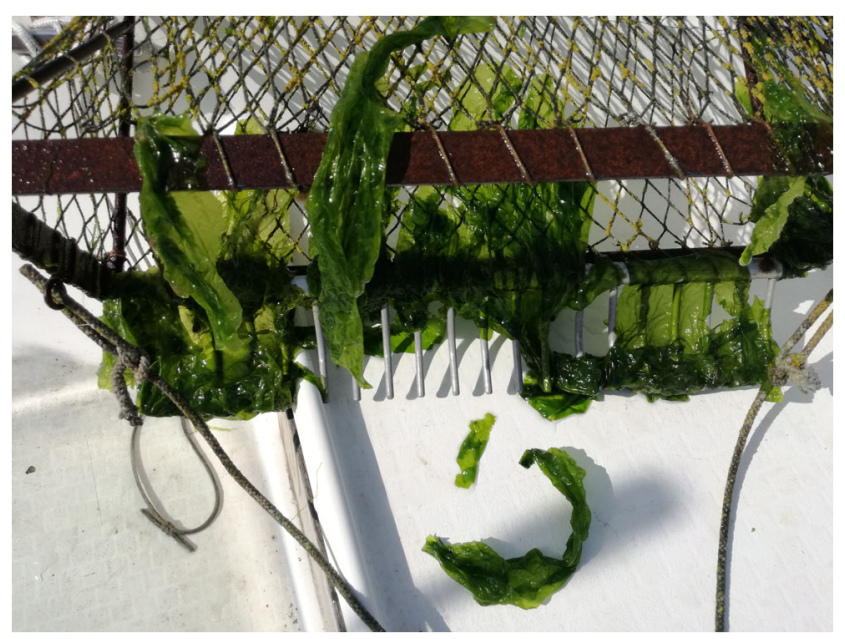

Fig. 4 Ulva detected in site 1
The MicaSense RedEdge-M camera is currently one of the most performant UAV multispectral cameras. It has five different dedicated sensors able to acquire infrared data in addition to the standard RGB and can be easily mounted on a DJI Phantom 3 Professional drone. Each sensor has a narrow-band filter for detecting data respectively in the blue, green, red, red edge, and near infrared wavelengths (Table 1). The global shutter allows to minimize all the issues related to the movement of the UAV during the flight.

Radiometric corrections to the raw digital numbers acquired may be further applied by the use of both a calibrated reflectance panel and a DLS. The former is a panel made of a material with an almost Lambertian behavior whose spectral characteristics have been determined by a calibration in laboratory. Conversely, the DLS is a sun sensor that measures the solar irradiance at the top of the aircraft and it is useful to take into account the change in the environmental light conditions as well as to normalize the data to standard conditions. This feature is particularly helpful in order to compare survey repetitions carried out in different environmental conditions. In the following the terms, DLS and sun sensor will be used indifferently.

The main advantages and disadvantages of radiometric calibration methods based on the use of a single device or both (DLS, known reflectance panel) are briefly summarized in Table 2, while in the following, those differences are discussed more in detail.

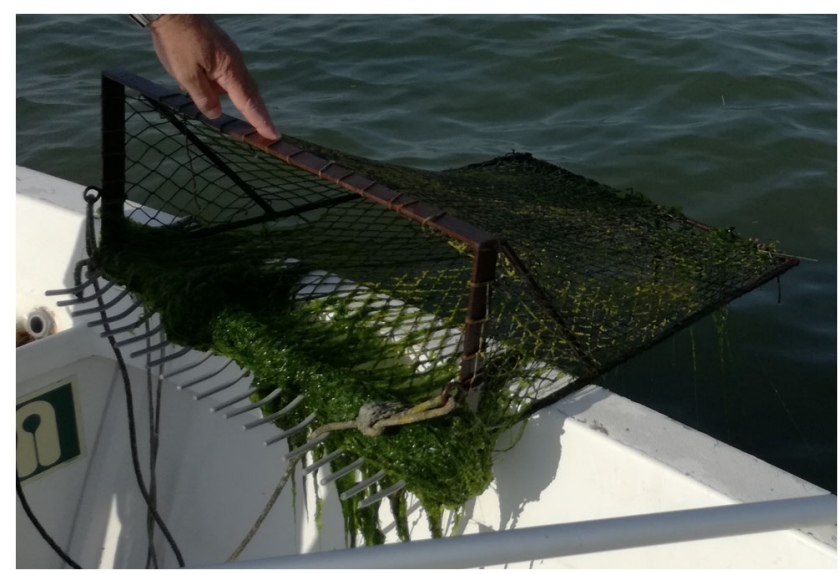

Fig. 5 Enteromorpha detected in site 2 


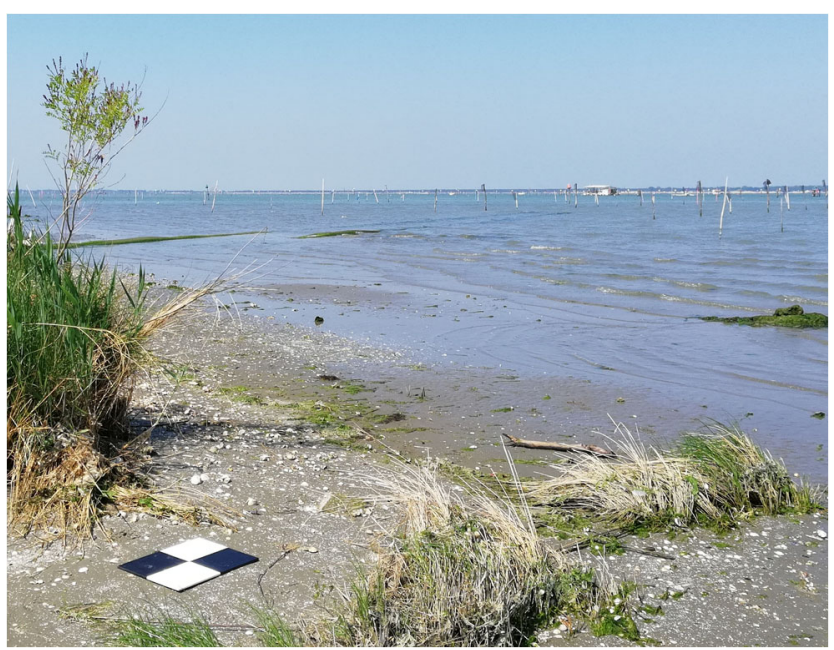

Fig. 6 Example of target used as ground control point

The combination of DLS irradiance measurements with the radiance detections made by the camera's sensors thus makes possible to compute reflectance values by the means of UAV's acquired imageries. The ratio between the reflected (camera measurements) and the incident radiation (DLS measurements) gives reflectance: the difference in the view angle of the sensors should be adequately considered since the DLS measures over the entire hemisphere, while the camera detects the radiance from a small view angle (Hakala et al. 2018).

In spite of the possibility to use DLS measurements to compute reflectance values, the use of a calibrated reflectance panel is certainly a more robust way to compute absolute reflectance values, especially whenever combined with the

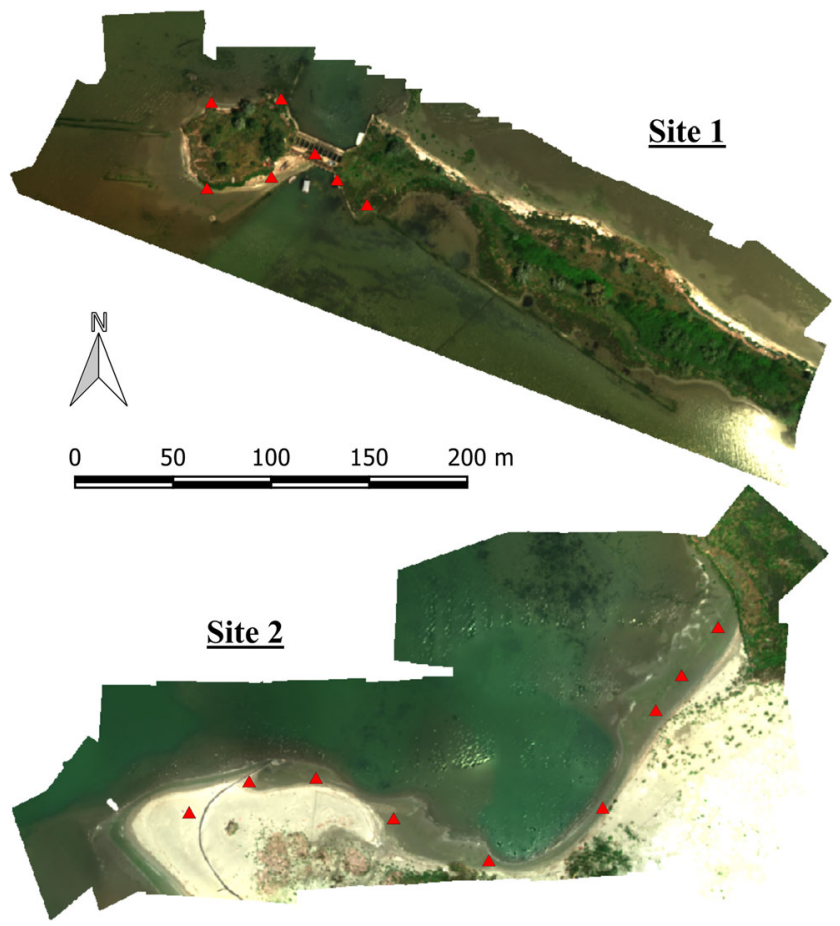

Fig. 7 Location of the targets used for the alignment of imageries

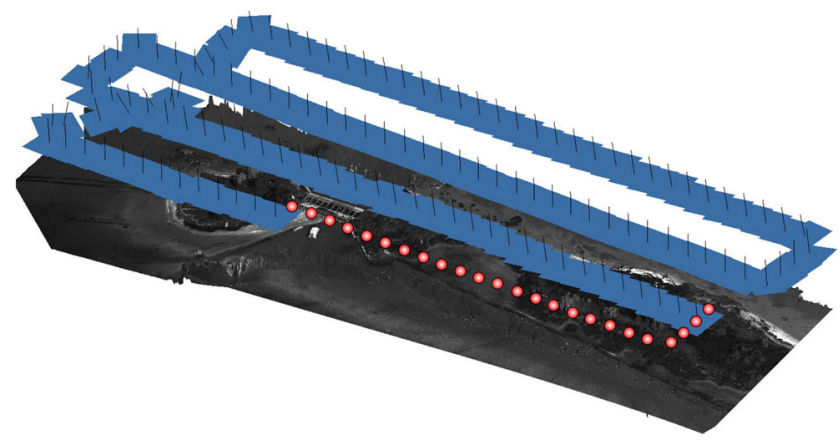

Fig. 8 Flight plan of the automatic mission in site 1. Red dots represent the images that could not be aligned due to the lack of reliable tie points

DLS data. This is because only using a panel it is directly possible to compute the relationship between radiance and reflectance on an image, in particular on the panel's portion of the image, and then further extend this relationship to the overall set of acquired imageries. In such a situation, the DLS data of each image may be considered in a differential way: it accounts for changes in the irradiance occurred from the time when the panel's image was captured and the time of every single image triggered during the UAV's flight.

In summary, the main advantage of the DLS by itself consists in allowing a continuous detection of the irradiance and it collects data whenever the multispectral camera is turned on. The direct interfacing with the camera makes possible to store the irradiance measurement performed at the time of image's capture within the Exif metadata in an automated way. Conversely, the reflectance computation gives less accurate results than using a known reflectance panel.

In the case that no DLS is available, the use of a known reflectance panel enables to compute reliable and precise reflectance values. However, the operator must remember to acquire an image of the panel without overexposing it, possibly just before and just after the flight. No changes in the irradiance during the flight may be taken into account without a DLS.

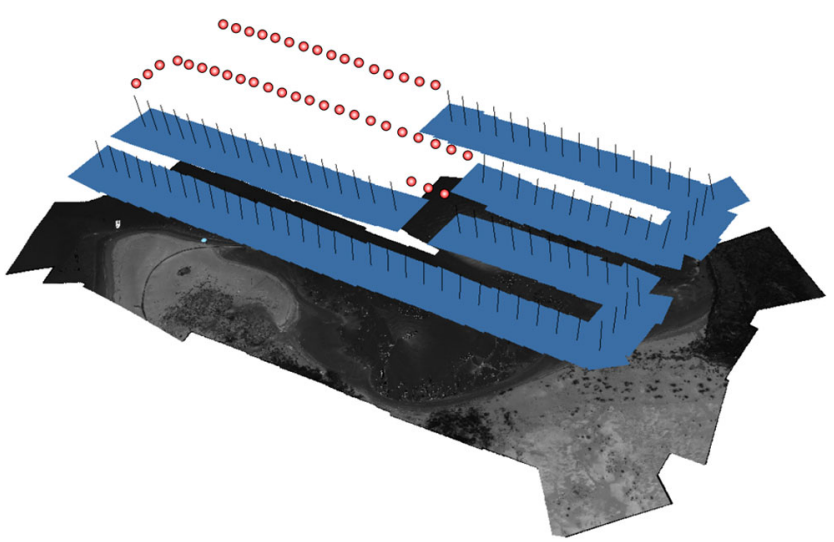

Fig. 9 Flight plan of the automatic mission in site 2. Red dots represent the images that could not be aligned due to the lack of reliable tie points 

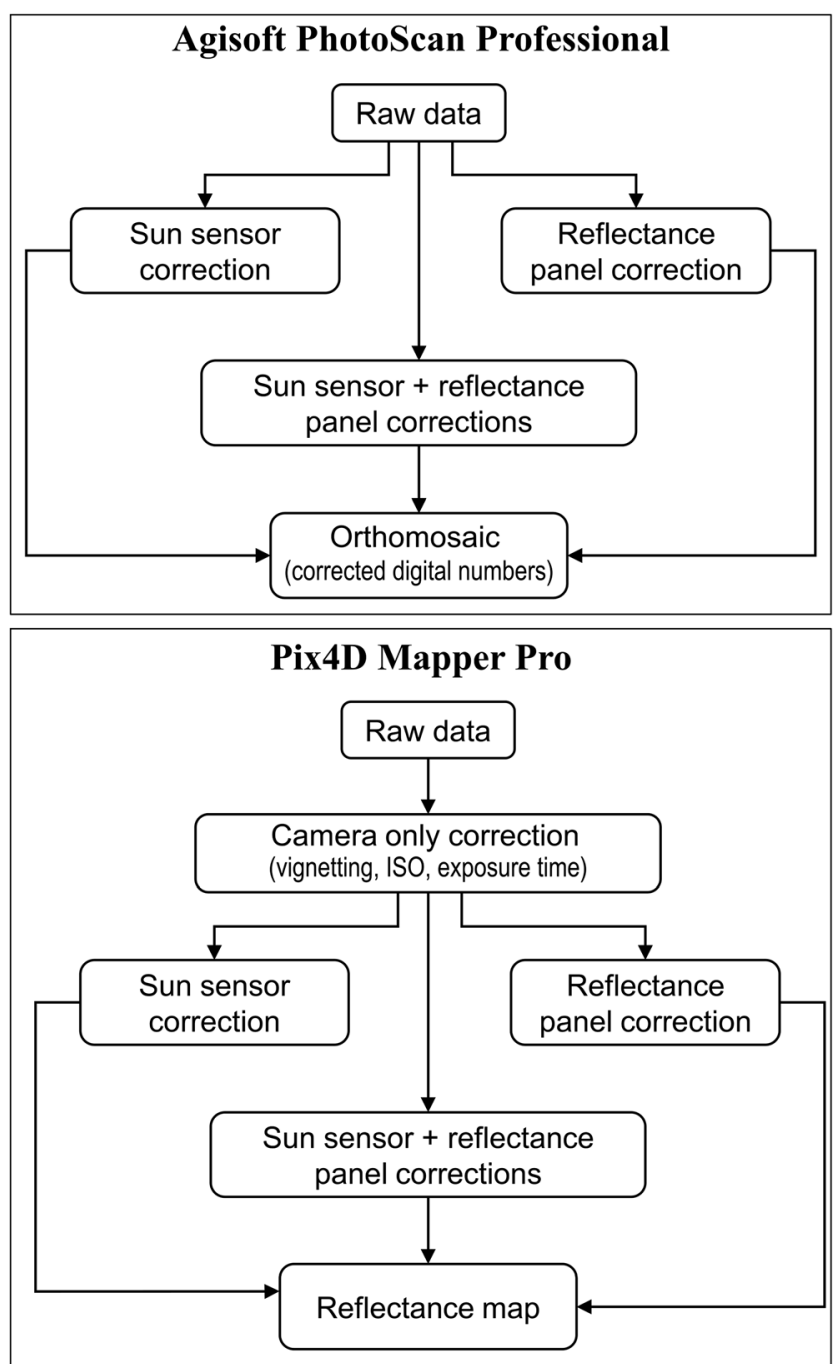

Fig. 10 Radiometric correction options for both the software used in the first part of the study

The combined use of a DLS and a known reflectance panel meets the advantages of the single approaches: accurate and reliable reflectance values can be computed and issues due to changes in the irradiance may be addressed by DLS measurements (e.g., transient clouds).

In addition to the DLS, the multispectral camera is also interfaced with a standard on-board GNSS that provides georeferencing information. Also in this case, the coordinates are stored within the Exif metadata of each single snapshot.

The case study area where the multispectral detections were performed included two different sites located at the end of the longest sandbar in the Southern part of the lagoon of Goro (Fig. 1). This area of the lagoon represented the most impacted by the eutrophication.

Two species of submerged seaweed were present in the selected sites: Ulva (Fig. 4) in site 1 and Enteromorpha in site 2 (Fig. 5).
The survey was carried out twice for each site. The first one, performed on May 25th, 2018, revealed the presence of submerged seaweed in an initial stage of its growth. The repetition, carried out on July 3rd, 2018, thus 39 days after the previous survey, pointed out a remarkable increase of the submerged seaweed, even though no significant emerged seaweed was present, with the only exception of the washed up one.

In order to survey each site, an automatic flight mission was planned setting a $70 \mathrm{~m}$ altitude. This value was adopted to ensure a theoretical ground sample distance (GSD) of a little less than $5 \mathrm{~cm}$ with the MicaSense RedEdge-M. The MicaSense RedEdge-M camera was set up to capture images with a longitudinal overlap of $80 \%$ and a side overlap of $70 \%$. Even if this latter value actually refers to the RGB native camera of the DJI Phantom 3, since the distance between strips is autonomously computed by the flight planner algorithm on the basis of a specified longitudinal overlap, the actual side overlap for the multispectral camera did not differ so much from the value above.

Ground control points (GCPs) were placed on the ground by using targets (Fig. 6). The location of all the deployed targets is shown in Fig. 7. The non-optimal distribution of the GCPs in site 1 (due to accessibility issues) did not affect the preliminary assessment of different radiometric calibration strategies or the comparison of the seaweed mapping in May and July. In fact, the first issue was addressed by creating a single template project beforehand, on which the targets were specified on the images. Different radiometric correction methods were applied only afterwards. Similarly, the georeferencing of the orthomosaics finally proved to be accurate enough to allow the comparison of algal detections performed in May and July.

The GCPs were surveyed through a GNSS geodetic receiver in network real-time kinematic (NRTK) mode and framed within the Italian official Reference System ETRS89ETRF2000 (2008.0).

The accuracy achievable using the NRTK has the same order of magnitude of the root mean square error in the reconstruction of the geometry with the SfM (Figs. 8 and 9) using a GSD of about $5 \mathrm{~cm}$.

All the imageries were processed through both the Agisoft PhotoScan Professional and the Pix4D Mapper Pro software.

As regards the alignment of the images by SfM algorithms, we have found some important issues:

- the lack of tie points due to the homogeneity of the sea surface (above all in image without coastline);

- the reflection of the sunshine over the sea surface generates an over-exposed area on the digital images along each strip. Many tie points are detected within this reflection zone and matched. 
Fig. 11 Homogeneous regions identified for the analysis of the radiometric calibration effects

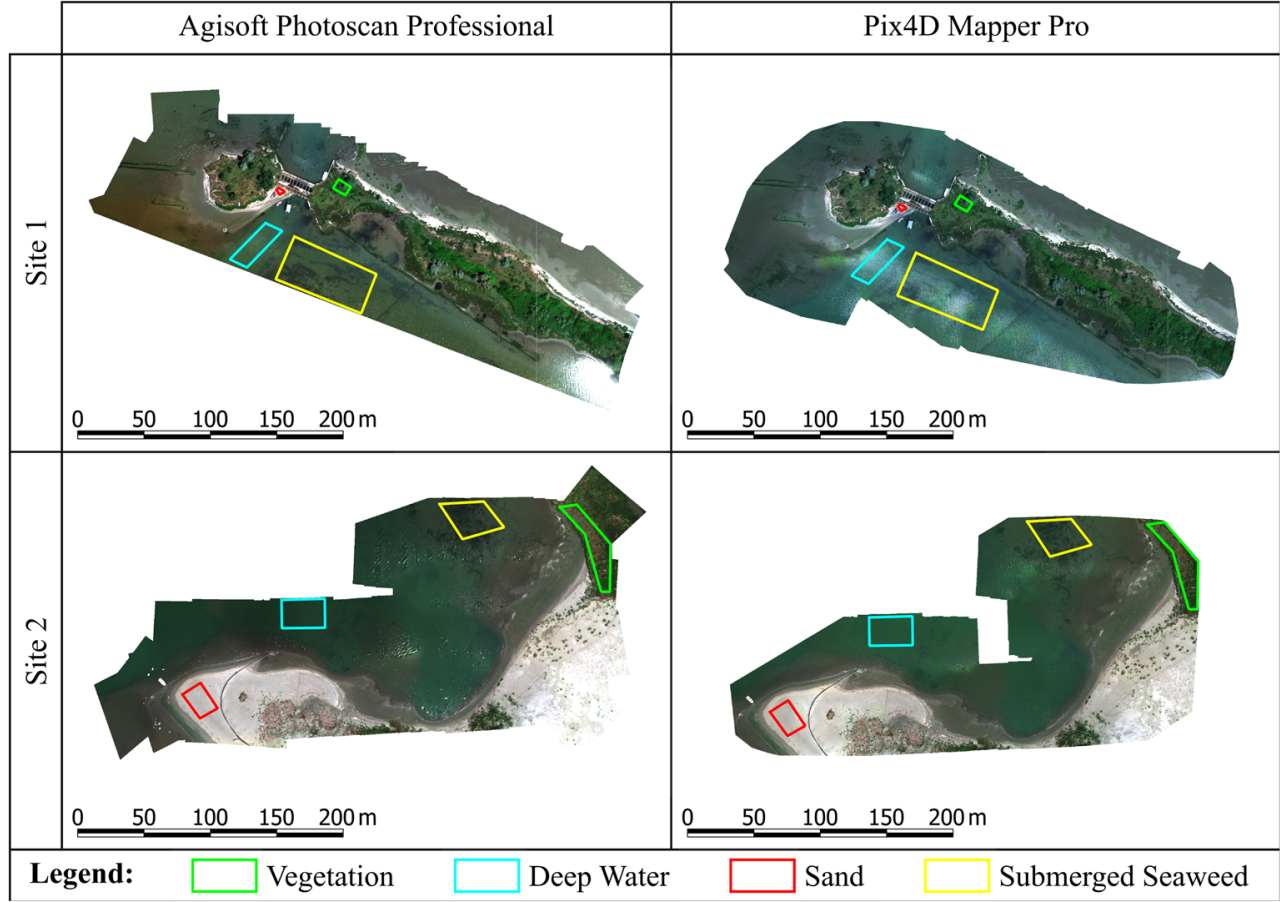

However, the outliers' detection algorithms were able to recognize these wrong matches.

Within each SfM software, a preliminary analysis on the effect of the different methods of radiometric calibration was performed. Imageries were hence processed by:

- applying no radiometric correction;

- applying only one calibration method (respectively, DLS and panel);

- applying both (DLS + panel).

The workflow summarizing the different combinations for both the software is shown in Fig. 10.

It is worth noting that through the Agisoft PhotoScan Professional, no reflectance value is ever obtained. Conversely, the output always consists in digital numbers within the theoretical range from 0 to 65,535 related to the 16-bit resolution of the input TIFF imageries. This applies to the calibrated output data as well. Using the Pix4D Mapper Pro, reflectance values are always generated whenever a correction is applied to the raw data. In addition, Pix4D Mapper Pro allows to compute the radiance from the DN with the correction option named "camera only". Therefore, Agisoft PhotoScan Professional finally generated orthomosaics only and never computed real reflectance maps, while Pix4D Mapper Pro could compute these latter ones in addition to the orthomosaics.

The final products that were considered in the preliminary assessment of radiometric calibration methods' effects in this study always consisted in the best type of output data retrievable by the software, thus orthomosaics whenever Agisoft PhotoScan Professional was used and reflectance maps for Pix4D Mapper Pro (with the only exception of the raw data).

For all the orthomosaics and the reflectance maps, the Normalized Difference Vegetation Index (NDVI) was computed from the data sensed in the red (R) and nearinfrared (NIR) wavelengths, through the well-known formula

$N D V I=\frac{N I R-R}{N I R+R}$

In order to investigate preliminarily and as accurately as possible whether a radiometric calibration would be really essential for a monitoring over time purpose, we identified four homogeneous areas in both test sites including not only the submerged seaweed (Fig. 11), respectively:

- the vegetation on the beach;

- the deep water (depth within the range $1 \div 1.5 \mathrm{~m}$ );

- the sand;

- the shallow water with submerged seaweed.

Each of the polygons defining the homogeneous areas was used as a mask for extracting the data in the blue, green, red, red edge, and near infrared narrow bands, as well as for the NDVI index synthetic band. Such operation was performed 
Fig. 12 Near Infrared values (digital number from orthomosaic) distribution for the four homogeneous regions: notice both the offset and the higher concentration of the values for all the classes due to the radiometric calibration, especially using the reflectance panel
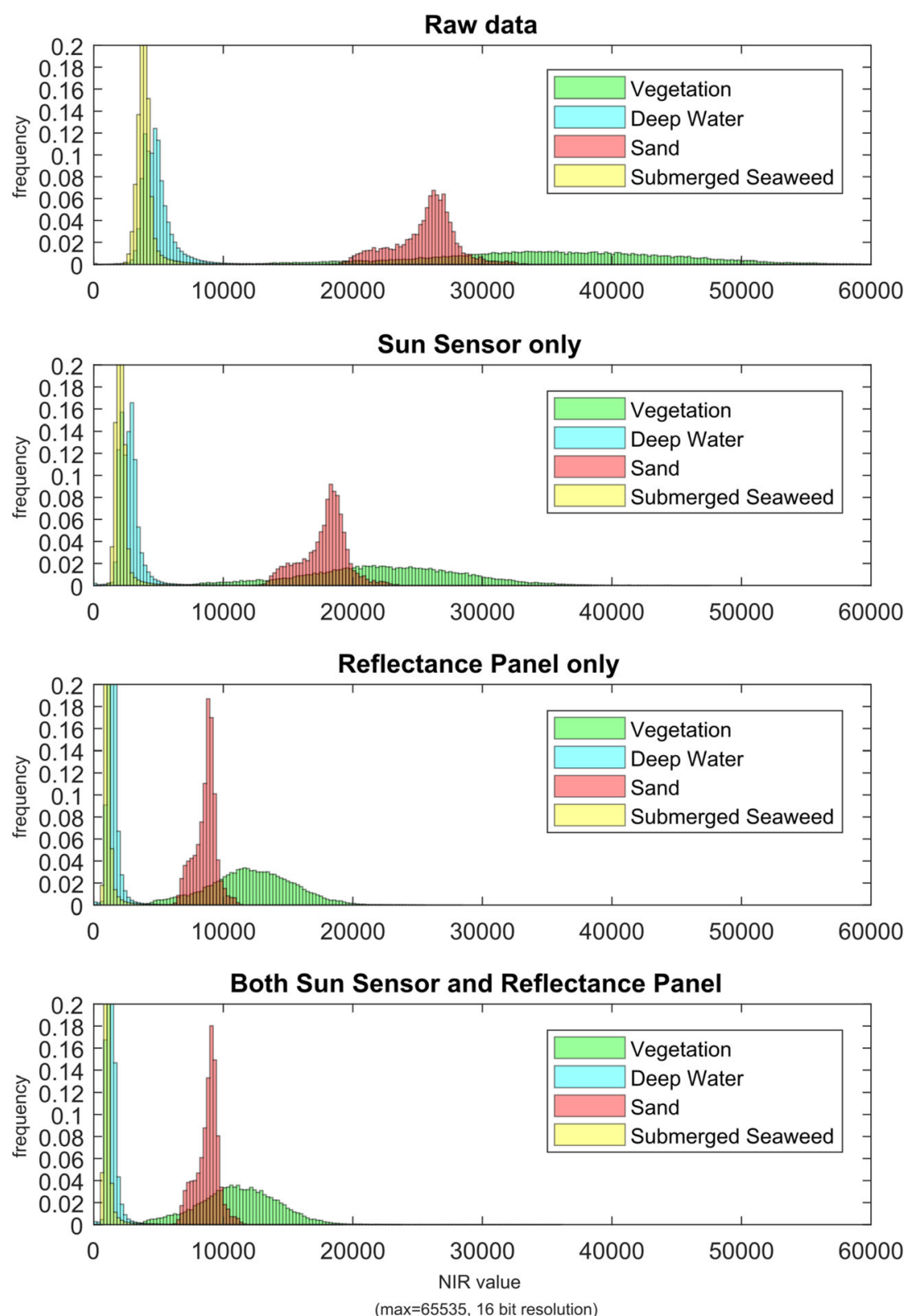

for any survey repetition (May 25th and July 3rd), for both the software used (Agisoft PhotoScan Professional and Pix4D Mapper Pro) and for all of the approaches of radiometric calibration of the raw data sensed (including the no-calibration option). A total of 36 different projects were created for this preliminary analysis.

All the orthomosaics and the reflectance maps were then processed through a MatLab® script for computing the frequency histograms of each class. The analysis was focused on the red, near infrared, and NDVI values. The results will be discussed further in the paper.

After the preliminary assessment of the effects of the different radiometric calibration procedures that could be undertaken with the MicaSense redEdge-M camera, the subsequent estimation of the submerged seaweed extents was thus solely performed on the radiometrically calibrated orthomosaics generated by using Agisoft PhotoScan Professional. In particular, two different approaches were investigated for the seaweed detection.

The first consisted in a simple NDVI computation in which a threshold value was assumed in order to distinguish between water pixels and seaweed pixels. The threshold value was assumed to be the same for the survey carried out in May and in July.

Conversely, the second one was a supervised classification with a maximum likelihood algorithm. In such approach, the operator needs to specify which land covers are recognizable on the orthomosaics by providing regions of interest (ROIs) 
Fig. 13 NDVI value distribution for the four homogeneous regions using Agisoft PhotoScan Professional: notice how any kind of radiometric calibration enables to distinguish between the many different non-vegetation pixels. This is crucial for classification purposes
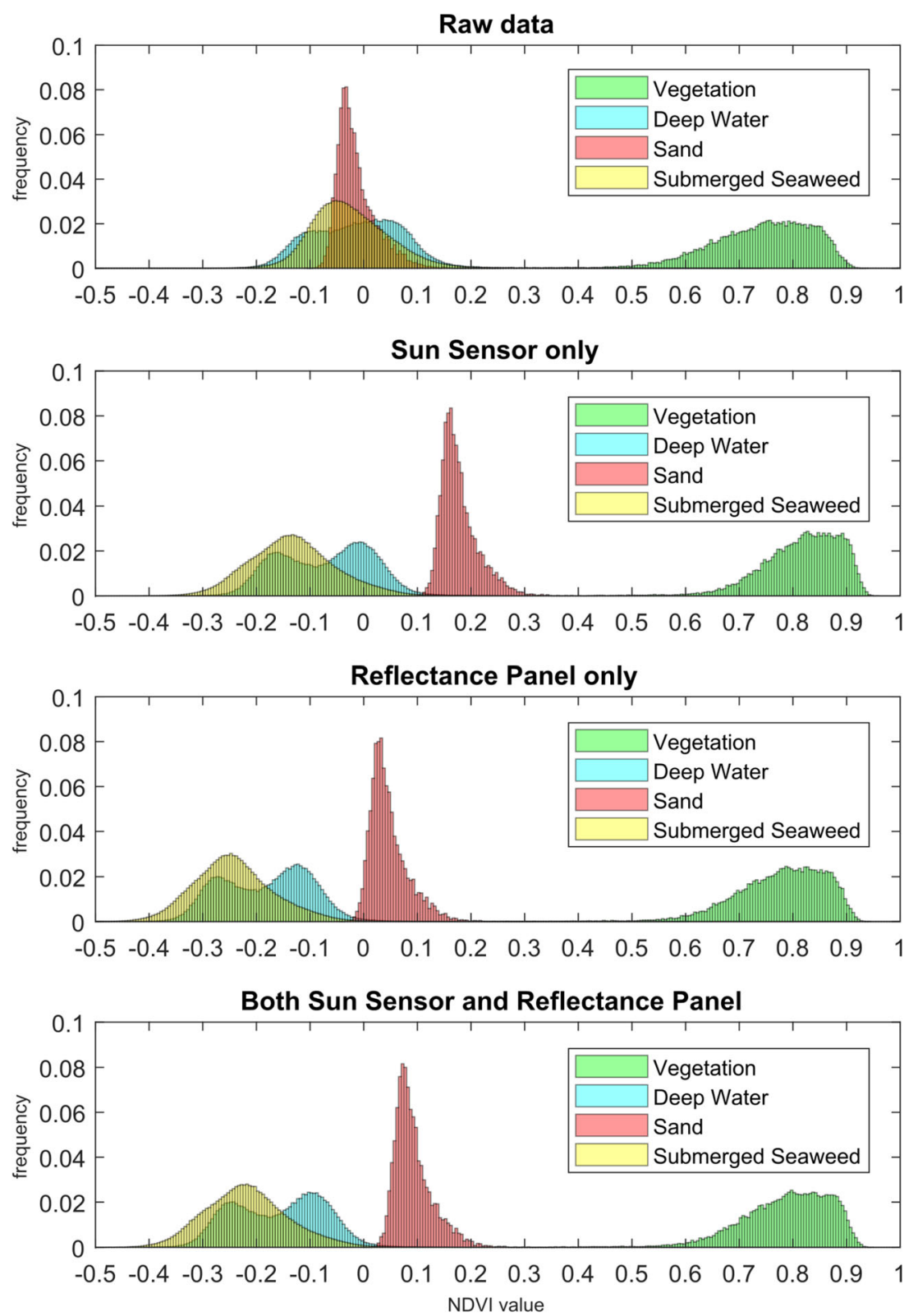

for the training. At the end, the accuracy of the classification can be assessed by the comparison of a further set of ROIs truth.

Finally, within this research, we also evaluated whether the additional information that the user provides by introducing ROIs may be useful to overcome the lack of a radiometric correction of the raw imageries. Therefore, a further supervised classification was conducted on the orthomosaics derived from the raw sensed imageries and these latters were compared with the results of the same classification performed on the radiometrically calibrated datasets. Once again, this investigation was focused on the purpose of generating an accurate and reliable submerged seaweed detection with the most possible simplified data processing.

\section{Results and discussion}

A preliminary analysis was performed on the distribution of the data values varying the radiometric calibration method. In particular, it was focused on the red and near infrared bands obtained in the final orthomosaics and reflectance maps extracted from the homogeneous regions described above (Fig. 11). Frequency histograms for each radiometric calibration option were generated. In addition, their combination in the form of the NDVI spectral index was also considered.

The frequency histogram contains the amount of pixels whose digital number (for orthomosaics) or reflectance (for reflectance maps) belongs to the considered interval. In order to make a consistent comparison between regions with a very different number of pixels, the frequencies were normalized. 
Fig. 14 NDVI value distribution for the four homogeneous regions using Pix4D Mapper Pro. Results are similar to the ones obtained through Agisoft PhotoScan Professional

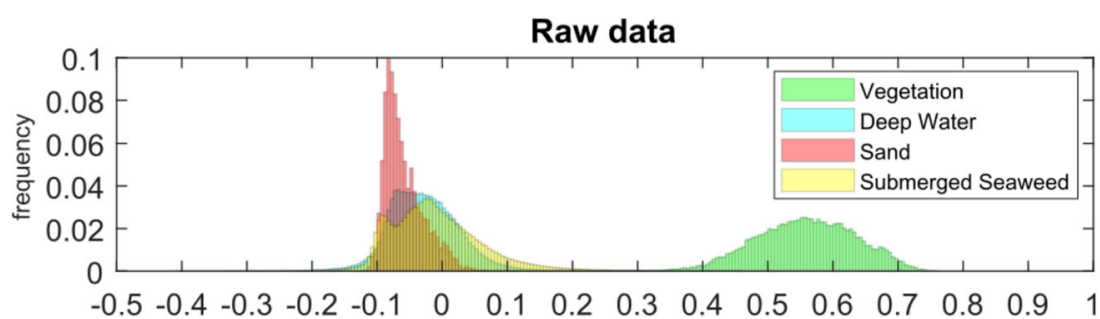

Camera only

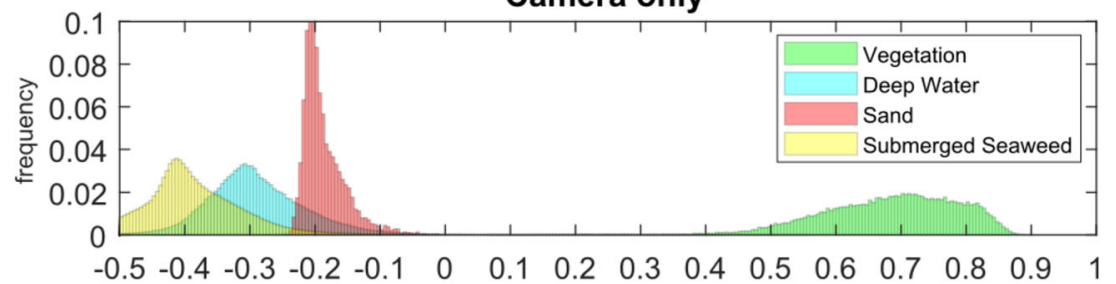

Camera + Sun Sensor

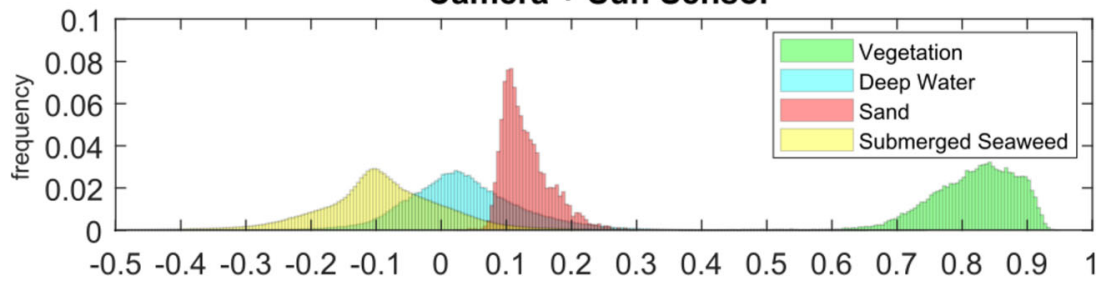

Camera + Reflectance Panel

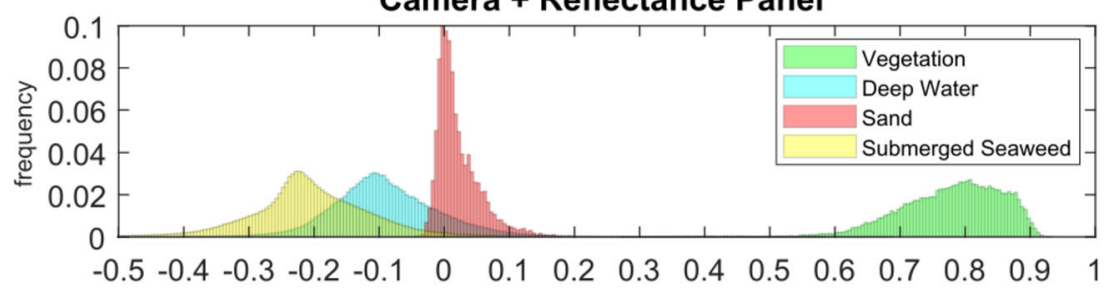

Camera + Sun Sensor and Reflectance Panel
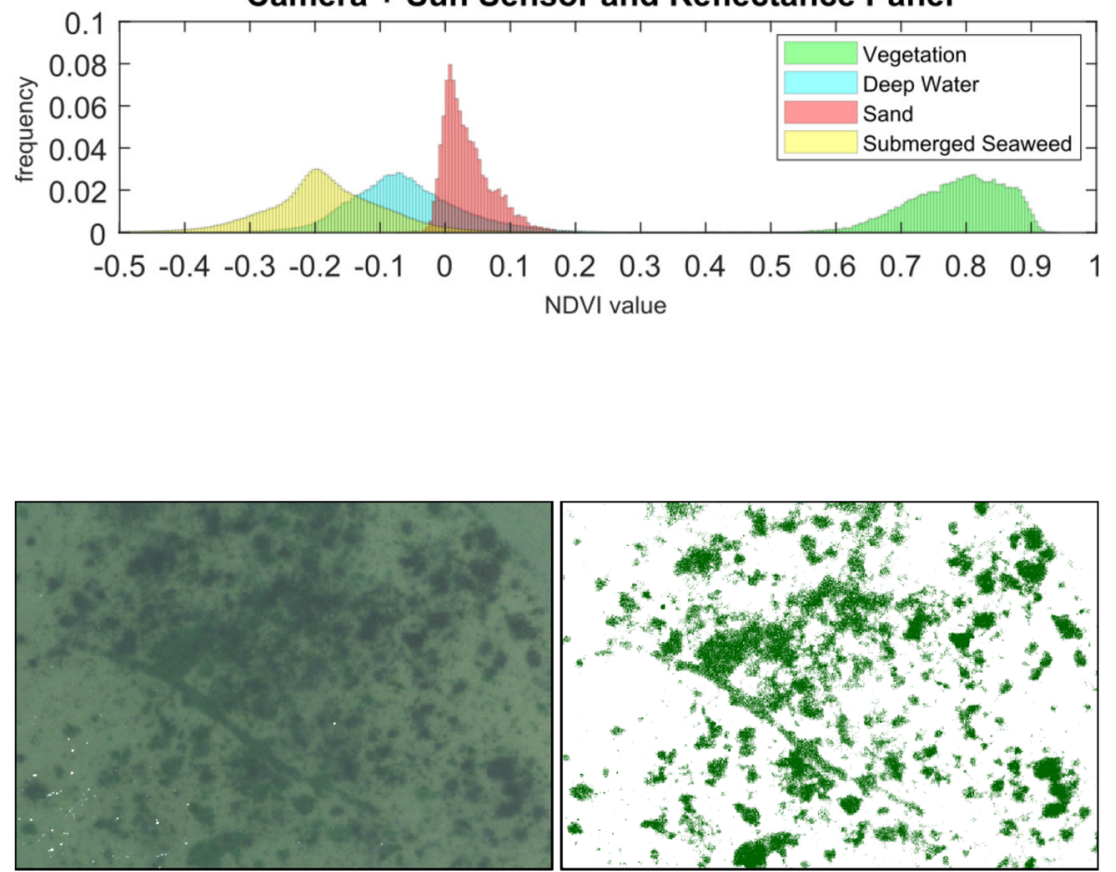

0

10

30

$40 \mathrm{~m}$ 


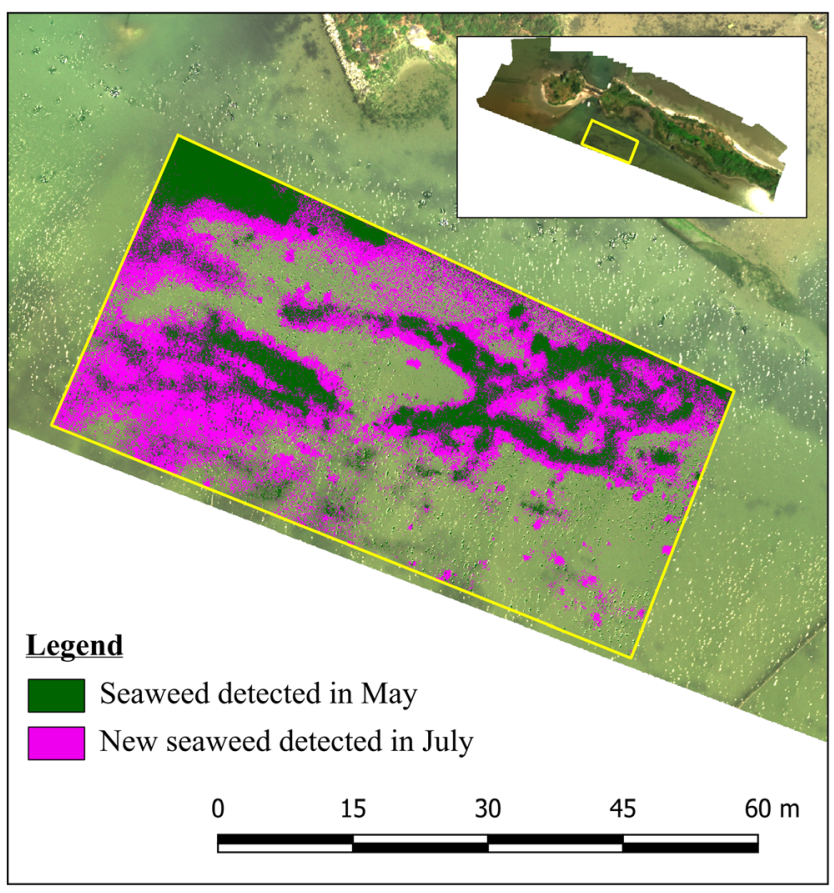

Fig. 16 NDVI-based detection of the seaweed within the sample polygon

First, it is worth noting that the application of any radiometric correction implies that the images, thus the resulting orthomosaics, become significantly darker (Fig. 12). This fact is due to the standardization to a reference ISO setting accounting also for the actual exposure time.

Secondarily, the comparison between the histograms of raw data and radiometric corrected data showed that only by applying a calibration, it is possible to distinguish among the different non-vegetation pixels based on the values of a spectral index. In particular, the NDVI

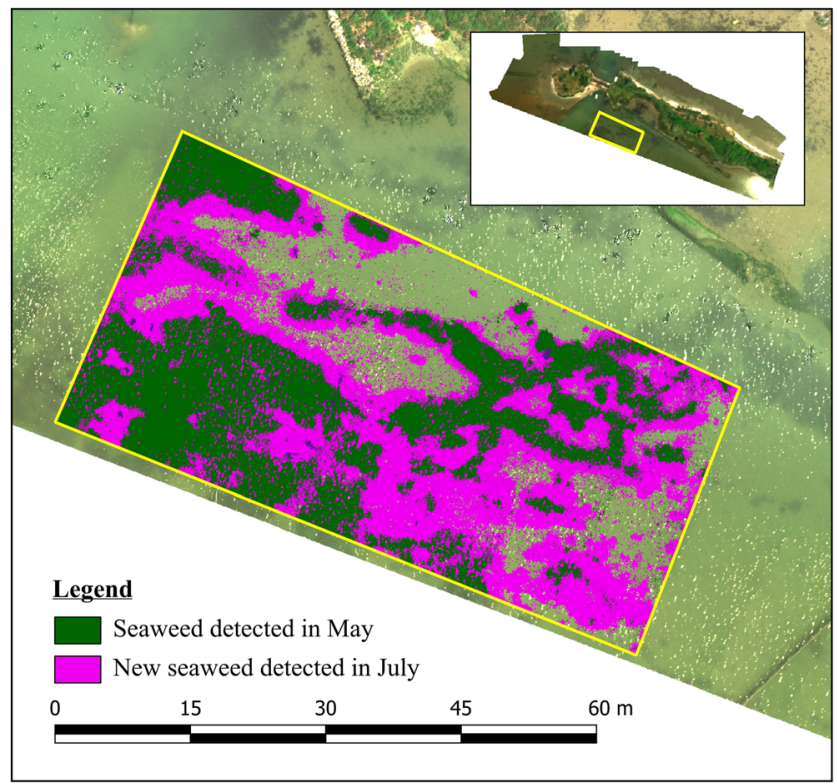

Fig. 17 Estimation of the seaweed's growth within the sample polygon through a supervised classification (maximum likelihood) computed by raw imageries did not allow to classify a pixel as a sand or a water with or without seaweed, while the same spectral index differentiated the sand from the water and the seaweed whenever any radiometric calibration method was applied (Figs. 13 and 14).

It is worth noting how the DLS data enables, on its own, to compute a correction for the data acquired in the narrow bands that already allows a separation in term of histograms' distributions. In fact, all the non-vegetation classes in Fig. 14 that have a high initial overlap tend to be more separated after the application of the simple DLS correction.

Even though the DLS itself allows the computation of the reflectance, it is only by using a known reflectance panel that the most accurate and reliable reflectance values may be computed. This is mainly due to the issue of a tilt of the DLS during the flight, as pointed out by Hakala et al. (2018). For such reasons, both the spectral data and the NDVI were found to be slightly different in our analysis whenever the known reflectance panel's images were considered or not.

Conversely, results generated either by using Agisoft PhotoScan Professional (Fig. 13) or Pix4D Mapper Pro software (Fig. 14) were found to be very similar, with only slight differences due to the use of different algorithms by the software.

After the preliminary analysis for the assessment of radiometric calibrations methods' effects on homogeneous regions, the detection of the submerged seaweed was hence performed. For all the reasons above, the mapping was performed on the multispectral orthomosaics generated by the solely Agisoft PhotoScan Professional and by applying radiometric corrections based on both the DLS irradiance measurements and the known reflectance panel's images.

The first approach for the detection and monitoring over time of the seaweed growth consisted in exploiting the NDVI. This index, in fact, is generally used to distinguish between vegetated and non-vegetated areas; thus, it should be helpful for a basilar seaweed detection.

The first detection was conducted within the same polygon used to identify the submerged seaweed zone in the first site.

The NDVI-based seaweed detection strongly depended on the actual distribution of the index values over the detected area. The analysis of the NDVI distribution revealed that the mean value was -0.22 in May while it rose to -0.15 in July, where negative values are due to the water absorption in the near infrared wavelengths.

However, no automatic procedure was used for this purpose and consequently the threshold was evaluated by changing the value and assessing the corresponding change in the detection of both surveys (May and July), also using the RGB visualization of the multispectral orthomosaic for reference (Fig. 15). This represented an unavoidable element of subjectivity related to the adoption of one of the simplest possible approaches, as it was for the NDVI-based estimation. 
Fig. 18 Estimation of the seaweed's expansion for site 1 within a wider area characterized by shallow water with variable water depth through a supervised classification (maximum likelihood)

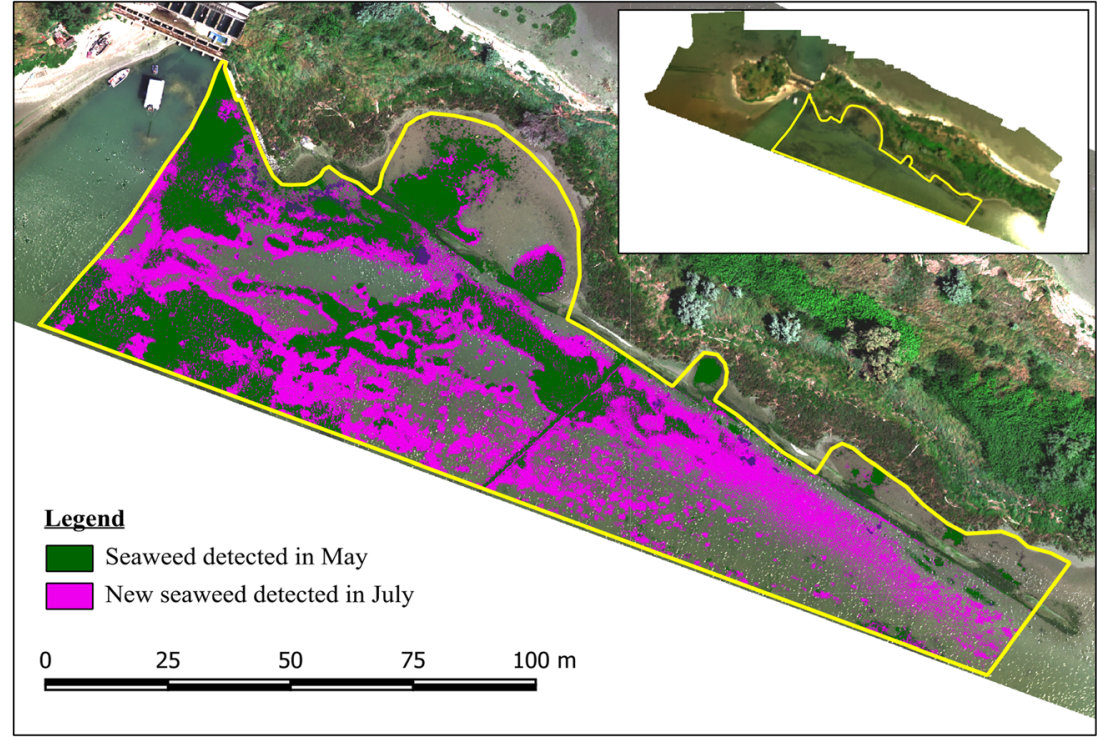

Finally, the threshold was set to -0.16 : values higher than this latter were classified as submerged seaweed, while the others represented water pixels.

The results of the estimation (Fig. 16) showed that the seaweed cover rose from 498.2 to $1179.6 \mathrm{~m}^{2}$. The rate of the increase was thus $+137 \%$ in 39 days.

The ground truth here consisted in an algal raking that revealed the effective presence of submerged seaweed in this area (Fig. 4).

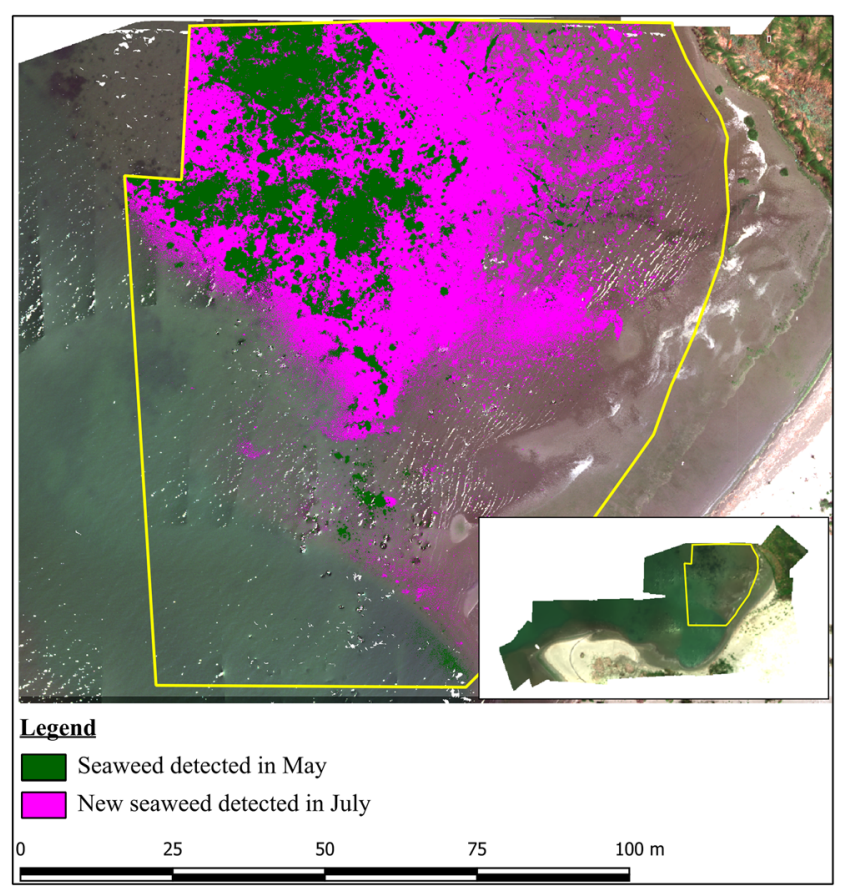

Fig. 19 Estimation of the seaweed's expansion for site 2 within a wide area characterized by shallow water with variable water depth through a supervised classification (maximum likelihood)
In order to produce a more reliable detection, a supervised classification was also performed as second approach. A maximum likelihood algorithm was used. Blue, green, red, red edge, and near infrared narrow bands were considered. In addition, the NDVI information was also taken into account as a sixth band. This approach pointed out an improvement in the reliability of the classification. ROIs were identified on each orthomosaic for every kind of land covers, including up to four different vegetation types, deep and shallow water, dry and wet sand, emerged and submerged seaweed.

For each ROI, a corresponding ROI truth was specified in order to assess the accuracy level of the classification. The computation of the confusion matrix allowed to calculate the overall accuracy (OA) (Richards and Xiuping 2006).

Every classification was validated by both evaluating that a high OA was finally obtained and also manually assessing the results through a comparison on the visible (RGB) orthomosaic. Similarly to what made for the NDVI approach, also in this case, an algal raking was performed to reveal the effective presence of the submerged seaweed.

The estimated submerged seaweed extent was $809.2 \mathrm{~m}^{2}$ in May and $1746.1 \mathrm{~m}^{2}$ in July; thus, the rate of increase with a supervised classification approach was $+116 \%$ (Fig. 17). It is possible to notice that the increment in terms of area extents is

Table 3 Feedbacks of the seaweed detected on the radiometrically uncorrected orthomosaic by a comparison to the classification on the corrected orthomosaic

\begin{tabular}{lllll}
\hline & $\begin{array}{l}\text { Site 1 } \\
\text { May 2018 }\end{array}$ & $\begin{array}{l}\text { Site 1 July } \\
2018\end{array}$ & $\begin{array}{l}\text { Site 2 May } \\
2018\end{array}$ & $\begin{array}{l}\text { Site 2 July } \\
2018\end{array}$ \\
\hline Submerged seaweed & $38.7 \%$ & $55.3 \%$ & $42.6 \%$ & $34.0 \%$ \\
Water & $39.6 \%$ & $11.0 \%$ & $50.6 \%$ & $40.7 \%$ \\
Other & $21.7 \%$ & $33.7 \%$ & $6.9 \%$ & $25.4 \%$ \\
\hline
\end{tabular}




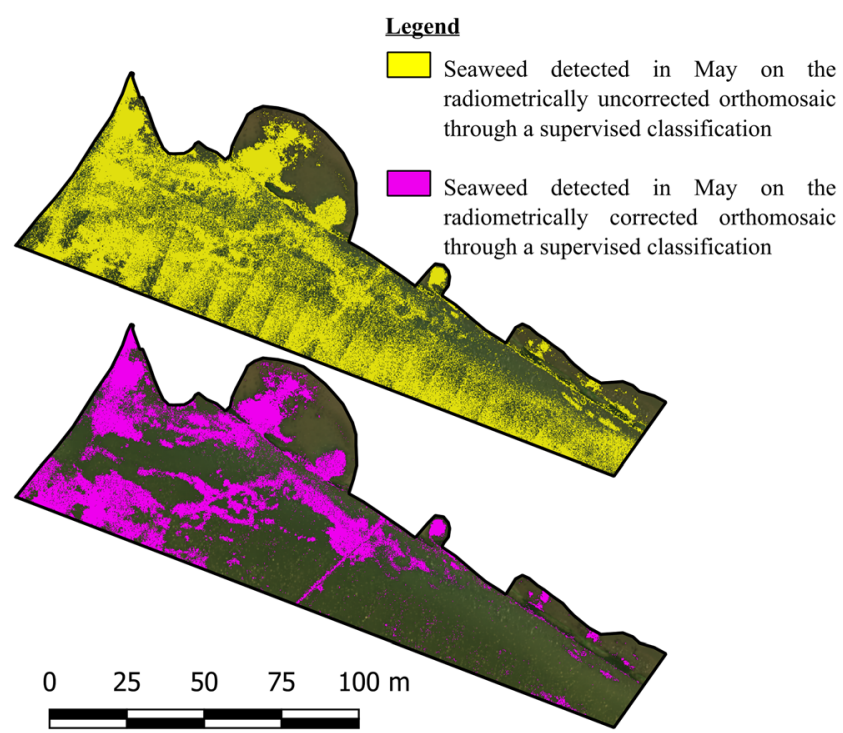

Fig. 20 Seaweed detections performed through a supervised classification using the same ROIs by both with and without the application of any radiometric correction to the raw data sensed for site 1

significantly higher if compared to the NDVI-based estimation, while the rate of increase is still comparable. Therefore, in a relative way the estimations are similar.

Both the approaches were thus extended outside the polygon. It is worth noting that the water depth was practically constant inside the considered polygon, while it was spatially variable (up to about $1.0 \mathrm{~m}$ ) in wider extents. For such reason, the detection performed through a supervised classification proved to be still reliable, while the NDVI-based detection failed due to the different water absorption in the near infrared at different water depth. In particular, the assumption of a unique and not depth-dependent threshold value for distinguish seaweed and water covers did not solve any detection task.

The results of the estimation (Fig. 18), supported and validated on the RGB visualization of the multispectral orthomosaic, showed that the seaweed cover rose from 2323.0 to $4908.6 \mathrm{~m}^{2}$ The rate of the increase was thus + $111 \%$ in 39 days.

On the basis of the best results obtained in site 1 (in terms of the most efficient technique to quantify the seaweed extents and expansion), the solely supervised classification approach was used in the second site.

The estimation (Fig. 19), supported by the validation conducted by both high OA values for the surveys (in May and in July) on the ROI truth and the comparison on the RGB orthomosaic, showed an increase of the seaweed cover from 1049.7 to $3558.1 \mathrm{~m}^{2}$. In addition, an algal raking was performed in site 2 and revealed the effective presence of submerged seaweed also in this area (Fig. 5). The rate of the increase was therefore $+239 \%$ in 39 days within the considered area.
Finally, after the successful mapping and monitoring over time of the submerged seaweed, a further supervised classification approach was investigated. The aim was to establish whether the supervised classification procedure could overcome the lack of radiometric corrections.

Therefore, the results of the classification obtained for the detection of the submerged seaweed shown above and a new classification performed on an orthomosaic generated by the dataset of the raw sensed (thus radiometrically uncorrected) imageries were compared.

It turned out that only a small fraction of the seaweed detected on the radiometrically uncorrected orthomosaics (within the range from 34.0 to $55.3 \%$ ) was found to be an actual seaweed cover after considering the DLS measurements and the known reflectance panel's images (Table 3 ).

In particular, this comparison (Fig. 20) was performed by computing how many pixels classified as a seaweed on the basis of raw imagery datasets were still classified in the same way using radiometrically corrected imageries. Practically, a confusion matrix was computed for the classification derived by the raw imageries and assuming the classification generated by calibrated imageries as a set of ROIs truth. Since those classification results were accurately validated beforehand, this assumption represented the most feasible hypothesis to perform a reliable comparison.

The use of raw imageries, without any radiometric calibration, provided an overestimation of the actual submerged seaweed extents. In addition, a general increase of the unclassified pixels was also recognized. The most probable explanation for those differences consists in the change in the light conditions for each image captured during the flight. This difference in the ISO setting and exposure time is also due to the high brightness of the sunshine reflection over the seawater that causes the overall image to be acquired significantly darker. The consequent effect when raw sensed images are merged together in an orthomosaic is hence an unbalanced image where the same land covers have significantly different digital numbers in all of the narrow bands.

\section{Conclusions}

The main purpose of this study was to test the use of multispectral lightweight cameras mounted on UAVs for the monitoring of the seaweed's growth in a shallow water coastal environment. In addition, as secondary purposes, we preliminarily assessed the effectiveness of the radiometric calibration computed and applied by two of the most common SfM software and we finally investigated whether a supervised classification may overcome the lack of radiometric corrections. 
With regard to the preliminary analysis, no significant differences were found between the results generated by the different software. The application of any kind of radiometric correction, also simply considering the DLS data only, enabled the distinction between non-vegetation classes. The evidence consisted in a separation of the frequency histograms.

The simplest technique tested for a quantification of the submerged seaweed growth was thus the use of the NDVI index by imposing a threshold value. A supervised classification through a maximum likelihood algorithm was also performed to compare the results. Even if strongly threshold-dependent, the NDVI quantification proved to be similar to the supervised classification one. However, the extension of this NDVI approach to a wider shallow water area was not possible because no unique threshold value could be found. The reason likely lies in the variable depth of the seawater that significantly affects the response of the seabed covers, especially in the near infrared, due to the water absorption in those wavelengths.

However, the supervised classification approach still gave reliable quantifications of the fraction of submerged seaweed for different sites at different epochs.

This latter result led to testing the real necessity of applying radiometric corrections. Therefore, a supervised classification (maximum likelihood) was performed on the overall orthomosaics respectively generated without the application of any kind of radiometric correction and considering both the DLS recorded data and the captured images of the known reflectance panel.

In this case, the results clearly showed how the classification performed on the radiometrically uncorrected orthomosaic was highly unreliable and strongly affected by issues related to the merging of images with different brightness. The application of an ISO sensitivity and exposure time normalization together with DLS data and images of a known reflectance panel allows to compensate for this.

All of the tests performed in this study confirm that the monitoring over time with a multispectral lightweight camera mounted on a UAV is possible. Moreover, this research highlights how the most reliable and accurate results can be achieved only by properly considering the application of radiometric corrections. Fortunately, this process is highly automatized in the most common commercial software such as Agisoft PhotoScan Professional and Pix4D Mapper Pro.

Acknowledgements The authors are grateful to the staff of the Regional Agency for Prevention, Environment and Energy of Emilia-Romagna (Arpae)-Oceanographic Structure Daphne for the support provided during the surveys in the field and some additional data regarding both the study sites and the lagoon of Goro.

The authors would also like to thank the graduate student Marco Miotto for his contribution.
Open Access This article is distributed under the terms of the Creative Commons Attribution 4.0 International License (http:// creativecommons.org/licenses/by/4.0/), which permits unrestricted use, distribution, and reproduction in any medium, provided you give appropriate credit to the original author(s) and the source, provide a link to the Creative Commons license, and indicate if changes were made.

\section{References}

Aasen H, Honkavaara E, Lucieer A, Zarco-Tejada PJ (2018) Quantitative remote sensing at ultra-high resolution with UAV spectroscopy: a review of sensor technology, measurement procedures, and data correction workflows. Remote Sens 10(7):1091. https://doi.org/10. 3390/rs10071091

Candiago S, Remondino F, De Giglio M, Dubbini M, Gattelli M (2015) Evaluation multispectral images and vegetation indices for precision farming applications from UAV images. Remote Sens 7:4026-4047. https://doi.org/10.3390/rs70404026

Corbau C, Munari C, Mistri M, Lovo S, Simeoni U (2016) Application of the principles of ICZM for restoring the Goro lagoon. Coast Manag 44(4):350-365

Deng L, Yan Y, Gong H, Duan F, Zhong R (2018a) The effect of spatial resolution on radiometric and geometric performances of a UAVmounted hyperspectral 2D imager. ISPRS J Photogramm Remote Sens 144:298-314

Deng L, Mao Z, Li X, Hu Z, Duan F, Yan Y (2018b) UAV-based multispectral remote sensing for precision agriculture: a comparison between different cameras. ISPRS J Photogramm Remote Sens 146: 124-136

Duffy JP, Pratt L, Anderson K, Land PE, Shutler JD (2018) Spatial assessment of intertidal seagrass meadows using optical imaging systems and a lightweight drone. Estuar Coast Shelf Sci 200:169-180

Hakala T, Markelin L, Honkavaara E, Scott B, Theocharous T, Nevalainen O, Näsi R, Suomalainen J, Viljanen N, Greenwell C, Fox N (2018) Direct reflectance measurements from drones: sensor absolute radiometric calibration and system tests for Forest reflectance characterization. Sensors 18(5):1417. https://doi.org/10.3390/ s18051417

Hallegraeff GM (2003) Harmful algal blooms: a global review. In: Manual on harmful marine microalgae; monographs on oceanographic methodology series, vol 11. UNESCO Publishing, Paris, pp 25-49

Honkavaara E, Khoramshahi E (2018) Radiometric correction of close-range spectral image blocks captured using an unmanned aerial vehicle with a radiometric block adjustment. Remote Sens 10(2):256. https://doi.org/10.3390/rs10020256

Hossain MS, Bujang JS, Zakaria MH, Hascim M (2015) The application of remote sensing to seagrass ecosystems: an overview and future research prospects. Int J Remote Sens 36(1):61-113

Jhan JP, Rau JY, Huang CY (2016) Band-to-band registration and orthorectification of multilens/multispectral imagery: a case study of MiniMCA-12 acquired by a fixed-wing UAS. ISPRS J Photogramm Remote Sens 114:66-77

Kislik C, Dronova I, Kelly M (2018) UAVs in support of algal bloom research: a review of current applications and future opportunities. Drones 2:35. https://doi.org/10.3390/drones2040035

Mancini F, Dubbini M, Gattelli M, Stecchi F, Fabbri S, Gabbianelli G (2013) Using unmanned aerial vehicles (UAV) for high-resolution reconstruction of topography: the structure from motion approach on coastal environments. Remote Sens 5:6880-6898. https://doi. org/10.3390/rs5126880

MicaSense (2018) MicaSense RedEdge image processing tutorial 1, https://github.com/micasense/imageprocessing/blob/master/ MicaSense Image Processing Tutorial 1, accessed 8 April 2019 
Nex F, Remondino F (2014) UAV for 3D mapping applications: a review. Appl Geomatics 6(1):1-15. https://doi.org/10.1007/s12518-0130120-x

Papakonstantinou A, Topouzelis K, Pavlogeorgatos G (2016) Coastline zones identification and 3D coastal mapping using UAV spatial data. Int J Geo-Inf 5:75. https://doi.org/10.3390/ijgi5060075

Richards JA, Xiuping J (2006) Remote sensing digital image analysis: an introduction, Fourth edn. Springler-Verlag, Berlin, p 439

Taddia Y, Corbau C, Zambello E, Pellegrinelli A (2019) UAVs for structure-from-motion coastal monitoring: a case study to assess the evolution of embryo dunes over a two-year time frame in the
Po River Delta, Italy. Sensors 19(4):1717. https://doi.org/10.3390/ s19071717

von Bueren SK, Burkart A, Hueni A, Rascher U, Tuohy MP, Yule IJ (2015) Deploying four optical UAV-based sensors over grassland: challenges and limitations. Biogeosciences 12:163-175

Wicaksono P, Kumara ISW, Kamal M, Fauzan MA, Zhafarina Z, Nurswantoro DA, Yogyantoro RN (2017) Multispectral resampling of seagrass species spectra: WorldView-2, Quickbird, sentinel-2A, ASTER VNIR and Landsat 8 OLI. IOP Conf Ser: Earth Environ Sci 98:012039. https://doi.org/10.1088/1755-1315/98/1/012039 